\title{
Spontaneous Symmetry Breaking in 5D Conformally Invariant Gravity
}

\author{
Taeyoon Moon ${ }^{1,2}$ and Phillial $\mathrm{Oh}^{1}$ \\ ${ }^{1}$ Department of Physics and Institute of Basic Science, Sungkyunkwan University, Suwon 440-746, Republic of Korea \\ ${ }^{2}$ Institute of Basic Science and Department of Computer Simulation, Inje University, Gimhae 621-749, Republic of Korea \\ Correspondence should be addressed to Phillial Oh; ploh@skku.edu
}

Received 4 May 2016; Revised 27 June 2016; Accepted 28 June 2016

Academic Editor: Barun Majumder

Copyright (c) 2016 T. Moon and P. Oh. This is an open access article distributed under the Creative Commons Attribution License, which permits unrestricted use, distribution, and reproduction in any medium, provided the original work is properly cited. The publication of this article was funded by SCOAP ${ }^{3}$.

\begin{abstract}
We explore the possibility of the spontaneous symmetry breaking in 5D conformally invariant gravity, whose action consists of a scalar field nonminimally coupled to the curvature with its potential. Performing dimensional reduction via ADM decomposition, we find that the model allows an exact solution giving rise to the 4D Minkowski vacuum. Exploiting the conformal invariance with Gaussian warp factor, we show that it also admits a solution which implements the spontaneous breaking of conformal symmetry. We investigate its stability by performing the tensor perturbation and find the resulting system is described by the conformal quantum mechanics. Possible applications to the spontaneous symmetry breaking of time-translational symmetry along the dynamical fifth direction and the brane-world scenario are discussed.
\end{abstract}

\section{Introduction}

Conformal symmetry is an important idea which has appeared in diverse area of physics, and its application to gravity has started with the idea that conformally invariant gravity in four dimensions (4D) [1-4] might result in a unified description of gravity and electromagnetism. The Einstein-Hilbert action of general relativity is not conformally invariant. In realizing the conformal invariance of this, a conformal scalar field is necessary $[5,6]$ in order to compensate the conformal transformation of the metric, and a quartic potential for the scalar field can be allowed. Its higher dimensional extensions are straightforward. In five dimensions (5D), conformal symmetry can be preserved with a fractional power potential $[7,8]$ for the scalar field. So far, it seems that little attention has been paid to the 5D conformal gravity with its fractional power potential. Such a potential renders a perturbative approach inaccessible, but nonperturbative treatment may reveal novel aspects. One can also construct a conformally invariant gravity with the Weyl tensor via $R$-squared gravity, but we focus on the Einstein gravity with a conformal scalar.
If the scalar field spontaneously breaks the conformal invariance with a Planckian VEV, the theory reduces to the 4D Einstein gravity with a cosmological constant $[9,10]$. On the other hand, the spontaneous symmetry breaking of Lorentz symmetry [11] or gauge symmetry [12] in 5D braneworld scenario [13-16] was studied, but little is known in the context of 5D conformal gravity. In this paper, we explore $5 \mathrm{D}$ conformal gravity with a conformal scalar and investigate possible consequences in view of the spontaneous symmetry breaking.

Let us consider 5D conformal scalar action of the form

$$
\begin{aligned}
S= & \int d^{5} x \sqrt{-g}\left[\frac{1}{2} \xi \phi^{2} R-\frac{\omega}{2} g^{A B} D_{A} \phi D_{B} \phi-V(\phi)\right] \\
& +S_{m}
\end{aligned}
$$

where $R$ is the five-dimensional curvature scalar, $S_{m}$ is the action for some matter ${ }^{1}$, and $A, B$ run over $0,1,2,3,4$. Here, $\xi$ is a dimensionless parameter describing the nonminimal coupling of the scalar field to the spacetime curvature. Also a parameter $\omega= \pm 1$ with $+(-)$ corresponds to canonical (ghost) 
scalar. For $\omega= \pm 1$, the conformal invariance of action (1) without matter term forces $\xi$ to be

$$
\begin{aligned}
V(\phi) & =V_{0}|\phi|^{10 / 3}, \\
\xi & =\mp \frac{3}{16},
\end{aligned}
$$

where $V_{0}$ is a constant and the corresponding conformal transformation is given by

$$
\begin{aligned}
g_{A B} & \longrightarrow e^{2 \sigma(x)} g_{A B}, \\
\phi & \longrightarrow e^{-(3 / 2) \sigma(x)} \phi(x) .
\end{aligned}
$$

When $\omega=-1$, the conformal scalar has a negative kinetic energy term, but we regard it as a gauge artifact [17] which can be eliminated from the beginning through field redefinition. Even with no scalar field remaining after gauging away for both cases $(\omega= \pm 1)$, the physical mass scale can be set since the corresponding vacuum solution requires introduction of a scale which characterizes the conformal symmetry breaking. In $4 \mathrm{D}$ conformal gravity, it is known that the conformal symmetry can be spontaneously broken at electroweak $[10,18]$ or Planck $[9,10]$ scale. In all cases, the action (1) becomes 5D Einstein action with a cosmological constant by redefinition of the metric, $\hat{g}_{A B}=\phi^{4 / 3} g_{A B}$, but we stick to the above conformal form (1) of the action to argue with the spontaneous breakdown of the conformal symmetry.

The paper is organized as follows. In Section 2, we perform the dimensional reduction from five to four dimensions by using the ADM decomposition. In Section 3, we present exact solutions with four-dimensional Minkowski vacuum $\left(R_{(4)}=0\right)$ and check if they can give a spontaneous breaking of the conformal symmetry. In Section 4, the gravitational perturbation and their stability for the solutions are considered. In Section 5, we include the summary and discussions.

\section{Dimensional Reduction (5D to 4D)}

In order to derive the $4 \mathrm{D}$ action from the $5 \mathrm{D}$ conformal gravity (1), we make use of the following ADM decomposition where the metric in $5 \mathrm{D}$ can be written as ${ }^{2}$

$$
\begin{aligned}
d S^{2}= & g_{A B} d x^{A} d x^{B} \\
= & g_{\mu \nu}(x, y)\left(d x^{\mu}+N^{\mu} d y\right)\left(d x^{\nu}+N^{\nu} d y\right) \\
& +\epsilon N^{2}(x, y) d y^{2} .
\end{aligned}
$$

To describe the background solution, we go to the "comoving" gauge and choose $N^{\mu}=0$. In this case, we can recover our $4 \mathrm{D}$ spacetime by going onto a hypersurface $\Sigma_{y}: y=y_{0}=$ constant, which is orthogonal to the 5D unit vector:

$$
\begin{aligned}
\widehat{n}^{A} & =\frac{\delta_{4}^{A}}{N}, \\
n_{A} n^{A} & =\epsilon,
\end{aligned}
$$

along the extra dimension, and $g_{\mu \nu}$ can be interpreted as the metric of the $4 \mathrm{D}$ spacetime. Using the metric ansatz (4), one obtains

$$
\begin{aligned}
R^{(5)} & =R^{(4)}-2 \frac{\nabla^{2} N}{N}+\frac{\epsilon}{N^{2}}\left(\frac{\stackrel{*}{N} g^{\alpha \beta} \stackrel{*}{g}_{\alpha \beta}}{N}-g^{\alpha \beta^{* *}}{ }_{\alpha \beta}\right. \\
& \left.-\frac{3 \stackrel{*}{g}^{\alpha \beta *} \stackrel{g}{\alpha \beta}_{\alpha \beta}}{4}-\frac{\left(g^{\alpha \beta^{*}}{ }_{\alpha \beta}\right)^{2}}{4}\right),
\end{aligned}
$$

where the asterisk ${ }^{*}$ denotes the differentiation with respect to $y$ and $\nabla^{2}=\nabla_{\mu} \nabla^{\mu}$ is the four-dimensional Laplacian. Using this, we find that action (1) becomes

$$
\begin{aligned}
S= & \int d^{5} x \sqrt{-g^{(4)}} N\left[\frac{1}{2} \xi \phi^{2} R^{(4)}\right. \\
& +\epsilon \frac{\xi \phi^{2}}{8 N^{2}}\left(\stackrel{* \alpha \beta}{g} \stackrel{*}{g}_{\alpha \beta}+\left(g^{\alpha \beta^{*}}{ }_{\alpha \beta}\right)^{2}+\frac{8 \stackrel{*}{\phi}^{\alpha}}{\phi} g^{\alpha \beta^{*}}{ }_{\alpha \beta}\right) \\
& \left.-\xi \nabla^{2} \phi^{2}-\frac{\omega}{2} g^{\mu \nu} \nabla_{\mu} \phi \nabla_{\nu} \phi-\epsilon \frac{\omega}{2 N^{2}} \stackrel{* *}{\phi} \phi-V(\phi)\right] \\
& +S_{m} .
\end{aligned}
$$

One can check that the above action (7) is invariant with respect to four-dimensional diffeomorphism $x^{\mu} \rightarrow x^{\prime \mu} \equiv$ $x^{\prime \mu}(x)$ with $N^{\prime}\left(x^{\prime}, y\right)=N(x, y)$. It is also invariant under $y \rightarrow y^{\prime} \equiv y^{\prime}(y)$ and $N \rightarrow N^{\prime} \equiv\left(d y^{\prime} / d y\right)^{-1} N$ apart from the matter action.

Before going further, we would like to comment on the homogeneous solution to the equations of motion given in $5 \mathrm{D}$ conformal gravity (1) without matter term. To this end, we first consider the Einstein equation for action (1), whose form is given by

$$
\begin{aligned}
R_{A B} & -\frac{1}{2} g_{A B} R=T_{A B}, \\
T_{A B} & \\
= & \frac{1}{\xi \phi^{2}}\left(\partial_{A} \phi \partial_{B} \phi-\frac{1}{2} g_{A B} \partial^{C} \phi \partial_{C} \phi-V g_{A B}\right) \\
& \quad+\frac{1}{\phi^{2}}\left(D_{A} D_{B} \phi^{2}-g_{A B} \square^{(5)} \phi^{2}\right)
\end{aligned}
$$

and the scalar equation can be written as

$$
0=\omega \square^{(5)} \phi+\xi \phi R-V^{\prime}(\phi),
$$

where $D_{A}$ is $5 \mathrm{D}$ covariant derivative, $\square^{(5)} \equiv D_{A} D^{A}$, and the prime' ${ }^{\prime}$ denotes the differentiation with respect to $\phi$. One can 
easily check that the homogeneous solution to (8) and (10) is given by

$$
\begin{aligned}
R_{A B} & =\Lambda g_{A B}, \\
\phi & =\phi_{0}, \\
\Lambda & =\frac{2 V_{0}}{3 \xi}\left(\phi_{0}\right)^{4 / 3} .
\end{aligned}
$$

We note that this solution can be approached in diverse ways. Firstly, field redefinition $\widetilde{g}_{M N}=\left(\phi / \phi_{0}\right)^{4 / 3} g_{M N}$ necessitates introduction of scale, which leads to five-dimensional Planck mass $M_{5}=\xi \phi_{0}^{2 / 3}$. Secondly, solution (11) corresponds to a gauge fixed case $\left(\phi=\phi_{0}\right)$ through the conformal transformation (3). Lastly, it can be interpreted as a vacuum solution obtained when considering an effective potential $V_{\text {eff }}=$ $-\xi R \phi^{2} / 2+V_{0}|\phi|^{10 / 3}$ (we will study the effective potential $V_{\text {eff }}$ for details at the end of the next section). In all cases, conformal symmetry is spontaneously broken with a symmetry breaking scale $\sim \phi_{0} \neq 0$. In addition, they yield the physically equivalent results: de Sitter $V_{0} / \xi>0$ or anti-de Sitter $V_{0} / \xi<$ 0 . It is to be noticed that both cases are classically stable. Also there is a huge degeneracy of vacuum solutions due to conformal invariance such that if $\left(g_{A B}^{(0)}(x, y), \phi^{(0)}(x, y)\right)$ is a solution, then $\left(\widetilde{g}_{A B}=e^{2 \sigma(x, y)} g_{A B}^{(0)}, \widetilde{\phi}=e^{-(3 / 2) \sigma(x, y)} \phi^{(0)}\right)$ is also a solution for an arbitrary function $\sigma(x, y)$. In the next section, we will investigate the explicit solution form, starting from the reduced action (7) without matter term.

\section{Exact Solutions}

From action (7) without matter term, we find the equation of motion for $N$ as

$$
\begin{aligned}
& 0=\frac{\xi \phi^{2}}{2} R^{(4)} \\
& -\frac{\epsilon \xi \phi^{2}}{8 N^{2}}\left(\stackrel{* \alpha \beta}{g} \stackrel{*}{g}_{\alpha \beta}+\left(g^{\mu \nu} \stackrel{*}{g}_{\mu \nu}\right)^{2}+\frac{8 \stackrel{*}{\phi}}{\phi} g^{\alpha \beta} \stackrel{*}{g}_{\alpha \beta}\right) \\
& -\xi \nabla^{2} \phi^{2}-\frac{\omega}{2} g^{\mu \nu} \nabla_{\mu} \phi \nabla_{\nu} \phi+\frac{\epsilon \omega}{2 N^{2}} \stackrel{* *}{\phi} \phi-V(\phi)
\end{aligned}
$$

and the equation for four-dimensional metric $g^{\mu \nu}$ is given by

$$
\begin{aligned}
& \frac{1}{2} \xi \phi^{2}\left(R_{\mu \nu}^{(4)}-\frac{1}{2} g_{\mu \nu} R^{(4)}\right)=T_{\mu \nu}^{(1)}+T_{\mu \nu}^{(2)}+T_{\mu \nu}^{(3)}+T_{\mu \nu}^{(4)} \\
& +T_{\mu \nu}^{(5)} \\
& T_{\mu \nu}^{(1)}=\frac{\xi}{2 N}\left[\nabla_{\mu} \nabla_{\nu}\left(N \phi^{2}\right)-g_{\mu \nu} \nabla^{2}\left(N \phi^{2}\right)\right], \\
& T_{\mu \nu}^{(2)}=\frac{\epsilon \xi \phi^{2}}{8 N^{2}}\left[\frac{1}{2} \stackrel{{ }^{\alpha \beta}}{g} \stackrel{*}{g}_{\alpha \beta} g_{\mu \nu}+2 \frac{N}{\phi^{2}}\left(\frac{\phi^{2}}{N}\right) \stackrel{*}{g}_{\mu \nu}+2 \stackrel{*}{g}_{\mu \nu}\right. \\
& +g^{\alpha \beta} \stackrel{*}{g}_{\alpha \beta} \stackrel{*}{g}_{\mu \nu}+\stackrel{*}{g} \stackrel{g^{\beta}}{g} \stackrel{*}{\beta \mu}_{\nu \alpha}+g^{\alpha \beta} \stackrel{*}{g}_{\beta \nu} \stackrel{*}{g}_{\mu \alpha} \\
& -\frac{1}{2}\left(g^{\alpha \beta^{*}} \stackrel{g}{\alpha \beta}^{2} g_{\mu \nu}-2\left(g^{\alpha{ }^{*}} \stackrel{*}{\alpha \beta}_{\alpha \nu}\right) g_{\mu \nu}\right.
\end{aligned}
$$

$$
\begin{aligned}
& \left.-2 \frac{N}{\phi^{2}}\left(\frac{\phi^{2}}{N}\right) g^{\alpha \beta^{*}}{ }_{\alpha \beta} g_{\mu \nu}\right], \\
& T_{\mu \nu}^{(3)}=-\frac{\xi}{N}\left[\nabla_{(\mu} N \nabla_{\nu)} \phi^{2}-\frac{1}{2} g_{\mu \nu} \nabla_{\rho} N \nabla^{\rho} \phi^{2}\right], \\
& T_{\mu \nu}^{(4)}=\left[\frac{\omega}{2} \nabla_{\mu} \phi \nabla_{\nu} \phi-\frac{\omega}{4} g_{\mu \nu} \nabla_{\rho} \phi \nabla^{\rho} \phi-\frac{1}{2} V(\phi) g_{\mu \nu}\right], \\
& T_{\mu \nu}^{(5)}=-\frac{\epsilon}{N}\left[\xi\left(\frac{{ }^{*} \phi}{N}\right) g_{\mu \nu}+\frac{\omega}{4} \frac{\phi^{*}}{N} g_{\mu \nu}\right] .
\end{aligned}
$$

Also the equation of motion for the scalar field can be written as

$$
\begin{aligned}
0 & =\xi N \phi R^{(4)}-2 \xi \phi \nabla^{2} N+\frac{\epsilon \xi \phi}{4 N}\left(\stackrel{*}{g} \stackrel{*}{g}_{\alpha \beta}-\left(g^{\alpha \beta} \stackrel{*}{g}_{\alpha \beta}\right)^{2}\right. \\
& \left.-4\left(g^{\alpha \beta^{*}} \stackrel{*}{\alpha \beta}\right)+4 \frac{N}{N} g^{\alpha \beta^{*}}{ }_{\alpha \beta}\right)+N\left(\omega \nabla_{\mu} \nabla^{\mu} \phi\right. \\
& \left.+\frac{\omega}{N} \nabla_{\mu} N \nabla^{\mu} \phi-V^{\prime}(\phi)\right)+\frac{\epsilon \omega}{2 N}\left(g^{\alpha \beta^{*}} \stackrel{*}{\alpha \beta}^{*}-2 \frac{N^{*} \phi}{N}\right. \\
& +2 \stackrel{* *}{\phi}) .
\end{aligned}
$$

Now one can equate (12) and trace of (13) and then obtain

$$
\begin{aligned}
& V(\phi)=-\frac{\xi}{2} \nabla^{2} \phi^{2}-2 \frac{\xi}{N} \nabla_{\mu} N \nabla^{\nu} \phi^{2}-\frac{3 \xi \phi^{2}}{2 N} \nabla^{2} N \\
& -\frac{\epsilon}{2 N}\left[8 \xi\left(\frac{\phi^{*} \phi}{N}\right)+3 \omega \frac{{ }^{*} \phi}{N}\right]+\frac{\epsilon \xi \phi^{2}}{8 N^{2}}\left[-3 \stackrel{*}{{ }^{\alpha \beta}} \stackrel{\leftrightarrow}{g}_{\alpha \beta}\right.
\end{aligned}
$$

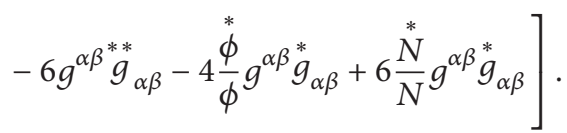

Substituting the above equation back into (12), we arrive at

$$
\begin{aligned}
& \frac{\xi \phi^{2}}{2} R^{(4)}=\frac{\xi}{2} \nabla^{2} \phi^{2}-2 \frac{\xi}{N} \nabla_{\mu} N \nabla^{\nu} \phi^{2}-\frac{3 \xi \phi^{2}}{2 N} \nabla^{2} N+\frac{\omega}{2} \\
& \cdot g^{\mu \nu} \nabla_{\mu} \phi \nabla_{\nu} \phi-\frac{2 \epsilon \omega^{*}}{N^{2}} \stackrel{*}{\phi}-\frac{4 \epsilon \xi}{N}\left(\frac{{ }^{*} \stackrel{*}{\phi}}{N}\right) \\
& +\frac{\epsilon \xi \phi^{2}}{8 N^{2}}\left(-2 \stackrel{*}{g} \stackrel{*}{g}_{\alpha \beta}+\left(g^{\alpha \beta} \stackrel{*}{g}_{\alpha \beta}\right)^{2}-6 g^{\alpha \beta^{* *}}{ }_{\alpha \beta}\right. \\
& \left.+\frac{4 \stackrel{*}{\phi}}{\phi} g^{\alpha \beta} \stackrel{*}{g}_{\alpha \beta}+\frac{6 \stackrel{*}{N}}{N} g^{\alpha \beta^{*}}{ }_{\alpha \beta}\right) \text {. }
\end{aligned}
$$


Let us focus on the conformally invariant case with $\omega=$ $-16 \xi / 3$ and $V(\phi)=V_{0}|\phi|^{10 / 3}$. In order to find solutions for this case, we consider the following ansatz:

$$
\begin{aligned}
g_{\mu \nu}(x, y) & =e^{-2 \mu^{2}\left(y-y_{0}\right)^{2}} \widehat{g}_{\mu \nu}(x), \\
N(x, y) & =N_{0} e^{-z_{1} \mu^{2} y^{2}}, \\
\phi(x, y) & =\phi_{0} e^{(3 / 2) z_{2} \mu^{2} y^{2}},
\end{aligned}
$$

where $N_{0}$ and $\phi_{0}$ are constants. For this ansatz, (15)-(17) become

$$
\begin{aligned}
& \frac{10}{3} V_{0} \phi_{0}^{7 / 3}=e^{2\left(z_{1}-z_{2}\right) \mu^{2} y^{2}} \frac{\phi_{0}}{N_{0}^{2}} \\
& \quad \epsilon \xi\left[80\left(1-z_{2}\right)\left(z_{1}-1\right) \mu^{4} y^{2}\right. \\
& \quad+80 z y_{0} y\left(2-z_{1}-z_{2}\right) \mu^{4}+40\left(1-z_{2}\right) \mu^{2} \\
& \left.\quad-80 y_{0}^{2} \mu^{4}\right], \\
& V_{0} \phi_{0}^{10 / 3}=e^{2\left(z_{1}-z_{2}\right) \mu^{2} y^{2}} \frac{\phi_{0}^{2}}{N_{0}^{2}} \\
& \quad \cdot \epsilon \xi\left[24\left(1-z_{2}\right)\left(z_{1}-1\right) \mu^{4} y^{2}\right. \\
& \quad+24 y_{0} y\left(2-z_{1}-z_{2}\right) \mu^{4}+12\left(1-z_{2}\right) \mu^{2} \\
& \left.\quad-24 y_{0}^{2} \mu^{4}\right], \\
& R^{(4)}=e^{2 z_{1} \mu^{2} y^{2}} \frac{2 \epsilon}{N_{0}^{2}}\left[24\left(1-z_{2}\right)\left(z_{1}-z_{2}\right) \mu^{4} y^{2}\right. \\
& \left.\quad-4 y_{0} y\left(z_{1}-z_{2}\right) \mu^{4}+12\left(1-z_{2}\right) \mu^{2}\right] .
\end{aligned}
$$

The above equation (21) determines $R^{(4)}$ as a function of $y$; namely, each hypersurface $y=\bar{y}$ has different values of $R^{(4)}$. But, we will restrict our attention to four-dimensional Minkowski space. Then, we notice that (19)-(21) always allow trivial vacuum solution $\phi_{0}=0, R^{(4)}=$ arbitrary, independent of $z_{1}$ and $z_{2}$, in general. The search for nontrivial vacuum with $\phi_{0} \neq 0$ is facilitated by the fact that the coefficients in (19) and (20) come out right so that the two equations are identical. Finally, for the 4D Minkowski vacuum $\left(R^{(4)}=0\right)$, we can obtain two solutions: (i) the r.h.s of (19)-(21) vanishes when $y_{0}=0$ and $z_{2}=1$, which yields $V_{0}=0, R^{(4)}=0$ and in this case, $z_{1}$ is arbitrary; (ii) for $V_{0} \neq 0$ and $y_{0} \neq 0$, they allow the solution of $z_{1}=z_{2}=1$. We summarize the $4 \mathrm{D}$ Minkowski solutions as follows:

$$
\text { (i) } \begin{aligned}
g_{\mu \nu} & =e^{-2 \mu^{2} y^{2}} \eta_{\mu \nu}, \\
N & =N_{0} e^{-z_{1} \mu^{2} y^{2}}, \\
\phi & =\phi_{0} e^{(3 / 2) \mu^{2} y^{2}}, \\
V_{0} & =0
\end{aligned}
$$

$$
\text { (ii) } \begin{aligned}
g_{\mu \nu} & =e^{-2 \mu^{2}\left(y-y_{0}\right)^{2}} \eta_{\mu \nu}, \\
N & =N_{0} e^{-\mu^{2} y^{2}}, \\
\phi & =\phi_{0} e^{(3 / 2) \mu^{2} y^{2}}, \\
V_{0} & =-24 \epsilon \xi \frac{y_{0}^{2} \mu^{4}}{N_{0}^{2} \phi_{0}^{4 / 3}} .
\end{aligned}
$$

Now we turn to an issue related to the spontaneous breaking of the conformal symmetry. We notice that the spontaneous symmetry breaking can be realized for a negative value of the curvature scalar with $R<0$ and $V_{0}>0$. To see this, we consider an effective potential $V_{\text {eff }}$ for the canonical scalar field $(\omega=1)$ and $V_{0}>0$ in action (1) as

$$
V_{\mathrm{eff}}=-\frac{1}{2} \xi \phi^{2} R+V_{0}|\phi|^{10 / 3} .
$$

As was mentioned in (2), here $\xi$ is fixed as a negative value of $\xi=-3 / 16$ for the canonical scalar $\omega=1$, which preserves the conformal symmetry of action (1). Since solution (i) $\left(V_{0}=\right.$ 0 ) with a stable equilibrium $\phi_{v}=0$ does not provide a symmetry-broken phase, we focus on the case (ii) with $\epsilon=1$ giving $V_{0}>0$ (hereafter we fix $\epsilon=1$ ).

It turns out that, for (ii) with the positive $5 \mathrm{D}$ curvature scalar $R>0$, we have only one vacuum solution of $\phi_{v}=0$, while for $R<0$, there exist two vacua of $\pm \phi_{v}$ with nonzero value:

$$
\phi_{v}=e^{(3 / 2) \mu^{2} y_{*}^{2}} \phi_{0}
$$

where $y_{*}$ is given by $y_{*}=4 y_{0} / 3 \pm \sqrt{3 / \mu^{2}+16 y_{0}^{2}} / 3$. In this case, the conformal symmetry is spontaneously broken with the symmetry breaking scale $\phi_{v}$ given by (24). This result is summarized in Figure 1 which shows that the effective potential with $R>0$ has only one minimum $\phi_{v}=$ 0 (a), while for $R<0$, it has two minima with $\pm \phi_{v}$ (b) which corresponds to the case of spontaneous breaking of the conformal symmetry.

\section{Tensor Perturbation}

In this section, we explore the stability of solutions (i), (ii) by performing a tensor fluctuation around the solutions. Note that the impact of conformal invariance shows up in the perturbation theory. One can always go to the unitary gauge and choose $\varphi=0$ in the scalar perturbation with $\phi=\phi_{0}+\varphi$. On the other hand, when $\omega / \xi \neq-16 / 3$, it is no longer possible to gauge away the fluctuation, and $\varphi$ is a dynamical field coupled to other fluctuations.

To investigate a tensor fluctuation around solutions (i), (ii), we need to consider the tensor perturbation of the metric as

$$
\begin{aligned}
d s^{2} & =a^{2}\left(\eta_{\mu \nu}+h_{\mu \nu}\right) d x^{\mu} d x^{\nu}+N^{2} d y^{2} \\
& =a^{2}\left[\left(\eta_{\mu \nu}+h_{\mu \nu}\right) d x^{\mu} d x^{\nu}+d z^{2}\right]
\end{aligned}
$$




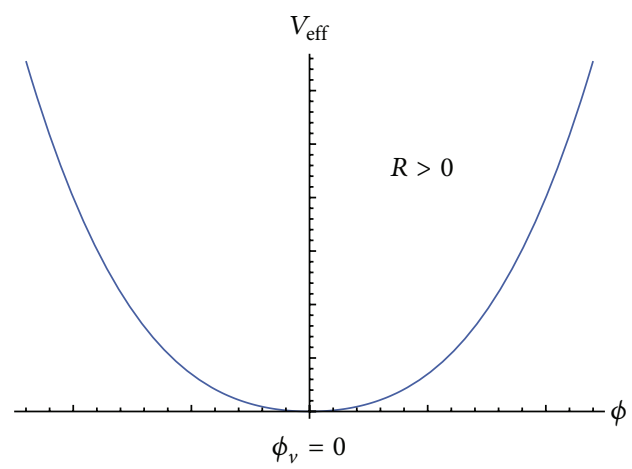

(a)

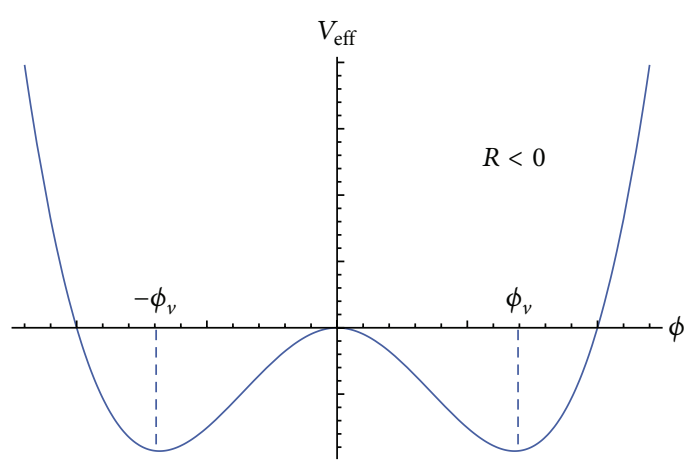

(b)

FIGURE 1: The effective potential $V_{\text {eff }}=-\xi R \phi^{2} / 2+V_{0}|\phi|^{10 / 3}$ with $\xi<0$ and $V_{0}>0$, where (a) [unbroken phase] corresponds to the effective potential with a positive value of the curvature scalar $R$, while (b) [broken phase] is a case of $R<0$.

where a new variable $z$ given in a conformally flat metric (26) satisfies $N d y=a d z$. It is found that the equation of motion for the tensor modes $h_{\mu \nu}$ is given by

$$
\partial_{z}^{2} h_{\mu \nu}+A(z) \partial_{z} h_{\mu \nu}-\square^{(4)} h_{\mu \nu}=0,
$$

where $h_{\mu \nu}$ satisfy the transverse-traceless gauge conditions $\left(\partial^{\mu} h_{\mu \nu}=0, h=\eta^{\mu \nu} h_{\mu \nu}=0\right)$ and $A(z)$ is given by

$$
A(z)=\frac{3}{a} \frac{\partial a}{\partial z}+\frac{2}{\phi} \frac{\partial \phi}{\partial z} .
$$

Consideration of a separation of variables $h_{\mu \nu}(x, z)=$ $f(z) H_{\mu \nu}(x)$ splits (27) into two parts:

$$
\begin{aligned}
{\left[-\partial_{z}^{2}+V_{\mathrm{QM}}\right] f } & =m^{2} f, \\
\square^{(4)} H_{\mu \nu} & =m^{2} H_{\mu \nu} .
\end{aligned}
$$

Here $m$ is the mass of four-dimensional Kaluza-Klein modes and the corresponding quantum mechanical potential $V_{\mathrm{QM}}$ reads

$$
V_{\mathrm{QM}}=\frac{1}{2} \frac{\partial A}{\partial z}+\frac{A^{2}}{4}
$$

One can check easily that $V_{\mathrm{QM}}$ vanishes for solution (i), which yields just plane wave solution with constant zero mode. On the other hand, for solution (ii), it gives an inverse square potential $^{3}$ as

$$
V_{\mathrm{QM}}=\frac{15}{4 z^{2}},
$$

where the corresponding Hamiltonian can be written as

$$
H=\frac{1}{2}\left(p_{z}^{2}+g z^{-2}\right) \quad \text { with } g=\frac{15}{4} .
$$

It is known in $[19,20]$ that, for the Schrödinger equation (29) with the inverse square potential $\left(V_{\mathrm{QM}}=g / z^{2}\right)$, the stability of the mode $f$ is determined by the condition ${ }^{4}$

$$
g>-\frac{1}{4}
$$

which guarantees that the graviton mode along the fifth dimension with $g=15 / 4$ is stable.

Before closing the section, we remark that the graviton mode along the fifth dimension preserves the residual conformal $S O(2,1)$ symmetry. It is well known that the quantum mechanical system of Hamiltonian (33) is conformally invariant [21], being referred to as conformal quantum mechanics (CQM). To see this, we first construct the CQM action for $H$ (33) from the Lagrangian formalism:

$$
S_{\mathrm{CQM}}=\frac{1}{2} \int d t\left(\dot{z}^{2}-\frac{g}{z^{2}}\right),
$$

which is invariant under the nonrelativistic conformal transformations:

$$
\begin{aligned}
& t^{\prime}=\frac{\alpha t+\beta}{\gamma t+\delta}, \\
& z^{\prime}=\frac{z}{\gamma t+\delta}
\end{aligned}
$$

with $\alpha \delta-\beta \gamma=1$.

In this case, it is known that three generators, that is, $H$ (time translation), $D$ (dilatation), and $K$ (special conformal) generators, can act with the transformation rules:

$$
\begin{aligned}
& H ; t^{\prime}=t+\tilde{t}, \\
& D ; t^{\prime}=\widetilde{d}^{2} t, \\
& K ; t^{\prime}=\frac{t}{\widetilde{k} t+1},
\end{aligned}
$$

where $\tilde{t}, \tilde{d}, \widetilde{k}$ are some constants and the generators $D$ and $K$ at $t=0$ in addition to $H(33)$ are given by

$$
\begin{aligned}
& D=-\frac{1}{4}\left(z p_{z}+p_{z} z\right), \\
& K=\frac{1}{2} z^{2} .
\end{aligned}
$$


These generators obey $\mathrm{SO}(2,1)$ commutations rules given by

$$
\begin{aligned}
& {[H, D]=i H,} \\
& {[K, D]=i K,} \\
& {[H, K]=2 i D,}
\end{aligned}
$$

whose Casimir invariant $\mathscr{C}$ is given by $\mathscr{C}=(H K+K H) / 2-$ $D^{2}=3 / 4$.

\section{Summary and Discussion}

In this paper, we considered the conformally invariant gravity in $5 \mathrm{D}$, which consists of a scalar field nonminimally coupled to the curvature with its potential. We found two solutions (i) and (ii) giving 4D Minkowski vacuum. By analyzing the dynamics of the metric perturbations around the solutions, we showed that two solutions are stable, since the former yields a plane wave solution with the constant zero mode, whereas the latter gives an inverse square potential. In particular, it was shown for solution (ii) that one has unbroken phase when $R>0, V_{0}>0$, while for $R<0, V_{0}>0$ the spontaneous breaking of the conformal symmetry can be realized with the scale $\phi_{v}$ given by (24).

We point out that solution (ii) may lead to a different mechanism which allows the possibility of a spontaneous breaking of translational invariance along the extra dimension. To this end, we consider a solution explicitly to (29) for $m^{2}=0$ as

$$
f(z)_{m^{2}=0}=c_{1} z^{5 / 2}+c_{2} z^{-3 / 2}
$$

with arbitrary constants $c_{1}$ and $c_{2}$. The first term of the r.h.s in (40) is not normalizable since the function $f(z)$ diverges at infinity, while the second term cannot lead to a normalizable solution due to its divergence at the origin. Thus, there is no normalizable zero mode solution. To resolve this problem, we define a new evolution operator $\mathscr{R}$ [21] given in terms of $K$ (38) and $H(33)$ :

$$
\mathscr{R} \equiv \frac{1}{2}\left(\frac{1}{a} K+a H\right),
$$

which yields the eigenvalues of $\mathscr{R}$ as follows:

$$
r_{n}=r_{0}+n, \quad r_{0}=\frac{3}{2}
$$

Here, $a$ is some constant with the length dimension. It turns out that the new evolution operator $\mathscr{R}(41)$ provides a normalizable ground state $f_{0}$ :

$$
f_{0}(z)=c_{0} z^{5 / 2} e^{-z^{2} / 2}, \quad z \geq 0
$$

where a constant $c_{0}$ is given by $c_{0}=1$, being obtained from the normalization condition $\int_{0}^{\infty}\left|f_{0}(z)\right|^{2} d z=1$. Importantly, even if we have the normalizable ground state (43) by introducing the new evolution operator $\mathscr{R}(41)$, it implements the spontaneous breaking of the conformal symmetry in the sense that the fundamental length scale $a$ is not included in the Lagrangian but generated by the particular form of the vacuum. On the other hand, it should be pointed out that since the well-defined ground state described by the Hamiltonian $H$ which generates the time translation is not present, it may lead to a spontaneous symmetry breaking of time-translational invariance along the dynamical fifth direction [22-25].

We conclude with the following remark. We see that both solutions (i) and (ii) can be characterized by Gaussian warp factor [26-28], where the maximum value is located at $y=$ 0 and $y=y_{0}$, respectively. But, the vacuum mode $\phi_{v}$ of (24) of the scalar field and the massless mode of gravity for the broken phase $\left(R<0, V_{0}>0\right)$ are not localized on the Gaussian brane, because a value of $y_{*}$ in (24) cannot be equivalent to $y_{0}$ and zero mode $(40)$ is written as $f(y)_{m^{2}=0}=$ $\widetilde{c}_{1} e^{-5 \mu^{2} y_{0} y}+\widetilde{c}_{2} e^{3 \mu^{2} y_{0} y}$ in $y$-coordinate. Thus, it seems that it is hard to describe the brane-world scenario with the current approach. One possible alternative to apply our result to the scenario would be to treat the conformal gravity discussed in this study (or its variation) as the conformal matter sector and introduce 5D Einstein-Hilbert action separately. Then, there could be a possibility of addressing some of the related issues, especially the brane stabilization as a consequence of the spontaneous symmetry breaking. The details will be reported elsewhere.

\section{Competing Interests}

The authors declare that they have no competing interests.

\section{Acknowledgments}

This work was supported by Basic Science Research Program through the National Research Foundation of Korea (NRF) funded by the Ministry of Education (Grant no. 2015R1D1A1A01056572).

\section{Endnotes}

1. We assume that the matter is confined on a hypersurface at $y=y_{m}$, where $y$ is the fifth coordinate. It is known that this matter in the brane has a geometrical origin in space-time-matter (STM) theory or induced-matter theory (IMT) [29-31]. Also, the IMT has been extended to the modified Brans-Dicke theory (MBDT) of type (1), where the induced matter exhibits interesting cosmological consequences $[32,33]$. However, in this paper we are interested in the phenomena of spontaneous symmetry breaking and, thus, we will be neglecting the matter sector.

2. $x^{\mu}$ are the coordinates in $4 \mathrm{D}$ and $y$ is the noncompact coordinate along the extra dimension (see $[32,33]$ and references therein). We use spacetime signature $(-,+$, ,,++ \pm ), while $\epsilon= \pm 1$ denotes spacelike or timelike extra dimension.

3. The inverse square potential (32) corresponds to a repulsion from the origin. For an attractive potential $(\alpha<-1 / 4)$ case with $V(x)=\alpha x^{-2}$, it was shown in 
[34] that the quantum mechanical system has infinite continuous bound states from negative infinity to zero.

4. This condition yields exactly the BF bound [35] given in the $d$-dimensional AdS spacetime for the one-dimensional Schödinger equation:

$$
\begin{aligned}
& -\partial_{x}^{2} \psi+\frac{m^{2}+\left(d^{2}-1\right) / 4}{x^{2}}=E \psi \longrightarrow m^{2} \geq m_{\mathrm{BF}}^{2} \\
& =-\frac{d^{2}}{4}
\end{aligned}
$$

when replacing $g$ with $m^{2}+\left(d^{2}-1\right) / 4$.

\section{References}

[1] H. Weyl, "Gravitation and electricity," Sitzungsberichte der Preussischen Akademie der Wissenschaften zu Berlin (Physikalisch-Mathematische), vol. 1918, p. 465, 1918.

[2] H. Weyl, "Eine neue Erweiterung der Relativitätstheorie," Annalen der Physik, vol. 364, no. 10, pp. 101-133, 1919.

[3] V. H. Weyl, "Eine neue erweiterung der relatiritästheorie," Surveys in High Energy Physics, vol. 5, no. 3, pp. 237-240, 1986.

[4] H. Weyl, "Eine neue erweiterung der relativitätstheorie," Annalen der Physik, vol. 364, no. 10, pp. 101-133, 1919.

[5] P. A. M. Dirac, "A new basis for cosmology," Proceedings of the Royal Society of London A, vol. 165, pp. 199-208, 1938.

[6] P. A. Dirac, "Long range forces and broken symmetries," Proceedings of the Royal Society. London. Series A. Mathematical, Physical and Engineering Sciences, vol. 333, pp. 403-418, 1973.

[7] R. Jackiw and S.-Y. Pi, "Tutorial on scale and conformal symmetries in diverse dimensions," Journal of Physics. A. Mathematical and Theoretical, vol. 44, no. 22, Article ID 223001, 2011.

[8] T. Moon, J. Lee, and P. Oh, "Conformal invariance in EinsteinCartan-Weyl space," Modern Physics Letters A, vol. 25, no. 37, pp. 3129-3143, 2010.

[9] T. Padmanabhan, "Conformal invariance, gravity and massive gauge theories," Classical and Quantum Gravity, vol. 2, no. 5, pp. L105-L108, 1985.

[10] D. A. Demir, "Nonlinearly realized local scale invariance: gravity and matter," Physics Letters B, vol. 584, no. 1-2, pp. 133140, 2004.

[11] O. Bertolami and C. Carvalho, "Lorentz symmetry derived from Lorentz violation in the bulk," Physical Review D, vol. 74, no. 8, Article ID 084020, 2006.

[12] O. Bertolami and C. Carvalho, "Spontaneous symmetry breaking in the bulk and the localization of fields on the brane," Physical Review D, vol. 76, no. 10, Article ID 104048, 13 pages, 2007.

[13] N. Arkani-Hamed, S. Dimopoulos, and G. Dvali, “The hierarchy problem and new dimensions at a millimeter," Physics Letters B, vol. 429, no. 3-4, pp. 263-272, 1998.

[14] L. Randall and R. Sundrum, "Large mass hierarchy from a small extra dimension," Physical Review Letters, vol. 83, no. 17, pp. 3370-3373, 1999.

[15] L. Randall and R. Sundrum, "An alternative to compactification," Physical Review Letters, vol. 83, no. 23, pp. 4690-4693, 1999.

[16] P. D. Mannheim, Branelocalized Gravity, World Scientific, Hackensack, NJ, USA, 2005.
[17] I. Bars, P. Steinhardt, and N. Turok, "Local conformal symmetry in physics and cosmology," Physical Review D, vol. 89, no. 4, Article ID 043515, 2014.

[18] H. Cheng, "Possible existence of Weyl's vector meson," Physical Review Letters, vol. 61, no. 19, p. 2182, 1988.

[19] F. Calogero, "Solution of a three-body problem in one dimension," Journal of Mathematical Physics, vol. 10, no. 12, p. 2191, 1969.

[20] S. Moroz, "Below the Breitenlohner-Freedman bound in the nonrelativistic AdS/CFT correspondence," Physical Review D, vol. 81, Article ID 066002, 2010.

[21] V. de Alfaro, S. Fubini, and G. Furlan, "Conformal invariance in quantum mechanics," Il Nuovo Cimento A, vol. 34, no. 4, pp. 569-612, 1976.

[22] S. Fubini, "A new approach to conformal invariant field theories," Il Nuovo Cimento A, vol. 34, no. 4, pp. 521-554, 1976.

[23] E. D’Hoker and R. Jackiw, "Space-translation breaking and compactification in the Liouville theory," Physical Review Letters, vol. 50, no. 22, pp. 1719-1722, 1983.

[24] C. Bernard, B. Lautrup, and E. Rabinovici, "Monte Carlo study of the breakdown of translational invariance in a Liouville model," Physics Letters B, vol. 134, no. 5, pp. 335-340, 1984.

[25] E. Rabinovici, "Spontaneous breaking of space-time symmetries," Lecture Notes in Physics, vol. 73, p. 573, 2008.

[26] E. E. Flanagan, S. H. H. Tye, and I. Wasserman, "Brane world models with bulk scalar fields," Physics Letters. B, vol. 522, no. 1-2, pp. 155-165, 2001.

[27] J. Alexandre and D. Yawitch, "Branon stabilization from fermion-induced radiative corrections," Physics Letters B, vol. 676, no. 4-5, pp. 184-187, 2009.

[28] I. Quiros and T. Matos, "Gaussian warp factor: towards a probabilistic interpretation of braneworlds," https://arxiv.org/abs/ 1210.7553.

[29] P. S. Wesson, Space-Time-Matter: Modern Kaluza-Klein Theory, World Scientific, Hackensack, NJ, USA, 2007.

[30] J. Ponce De Leon, "Equivalence between space-time-matter and brane-world theories," Modern Physics Letters A, vol. 16, no. 35, pp. 2291-2303, 2001.

[31] N. Doroud, S. M. M. Rasouli, and S. Jalalzadeh, "A class of cosmological solutions in induced matter theory with conformally flat bulk space," General Relativity and Gravitation, vol. 41, no. 11, pp. 2637-2656, 2009.

[32] S. M. M. Rasouli, M. Farhoudi, and P. V. Moniz, "Modified Brans-Dicke theory in arbitrary dimensions," Classical and Quantum Gravity, vol. 31, no. 11, Article ID 115002, 26 pages, 2014.

[33] S. M. M. Rasouli and P. V. Moniz, "Exact cosmological solutions in modified Brans-Dicke theory," Classical and Quantum Gravity, vol. 33, no. 3, Article ID 035006, 2016.

[34] K. M. Case, "Singular potentials," Physical Review Letters, vol. 80, pp. 797-806, 1950.

[35] P. Breitenlohner and D. Z. Freedman, "Positive energy in anti-de Sitter backgrounds and gauged extended supergravity," Physics Letters B, vol. 115, no. 3, pp. 197-201, 1982. 

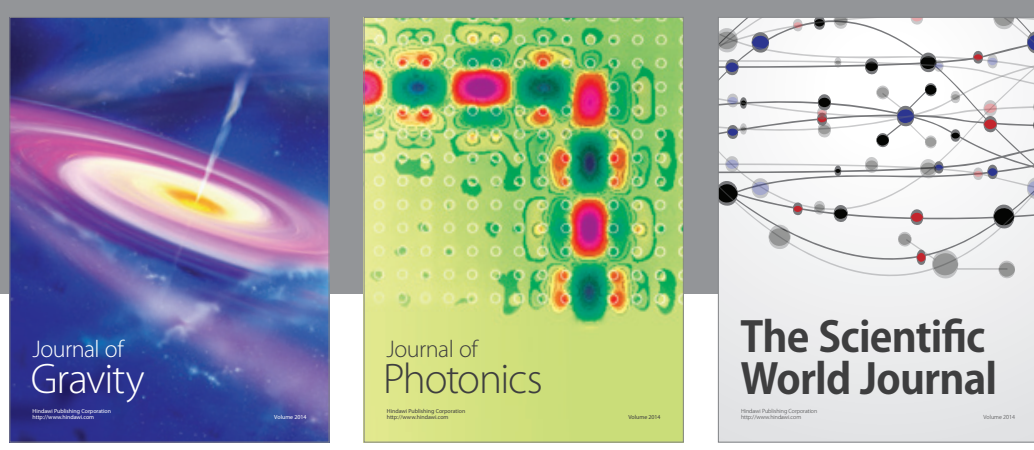

The Scientific World Journal
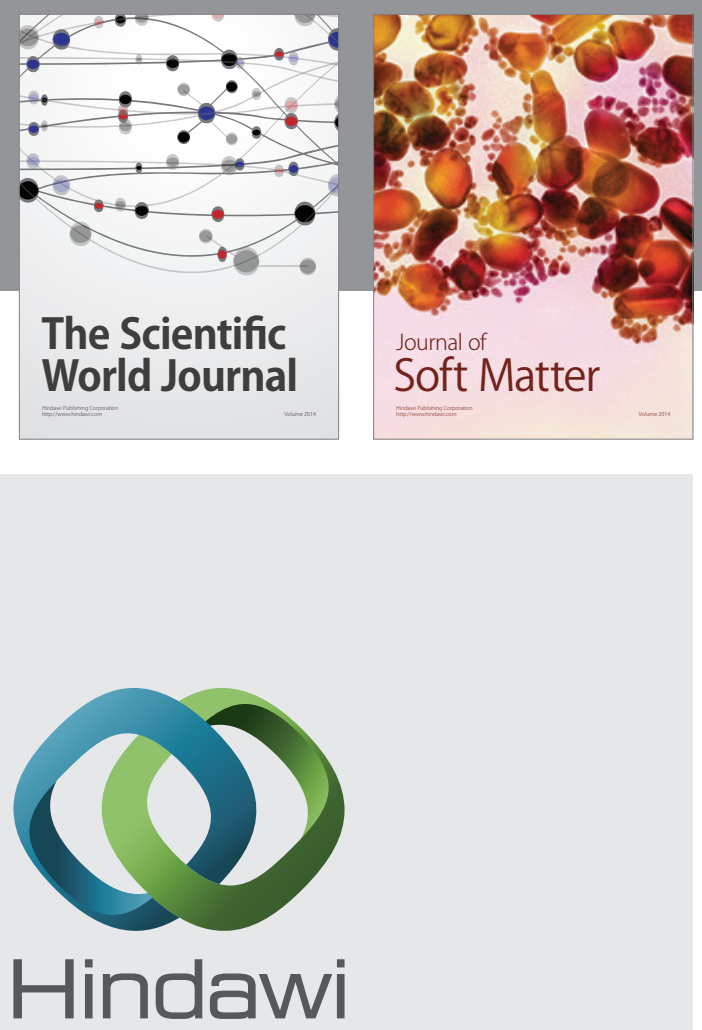

Submit your manuscripts at

http://www.hindawi.com

nternational Journal of

Statistical Mechanics
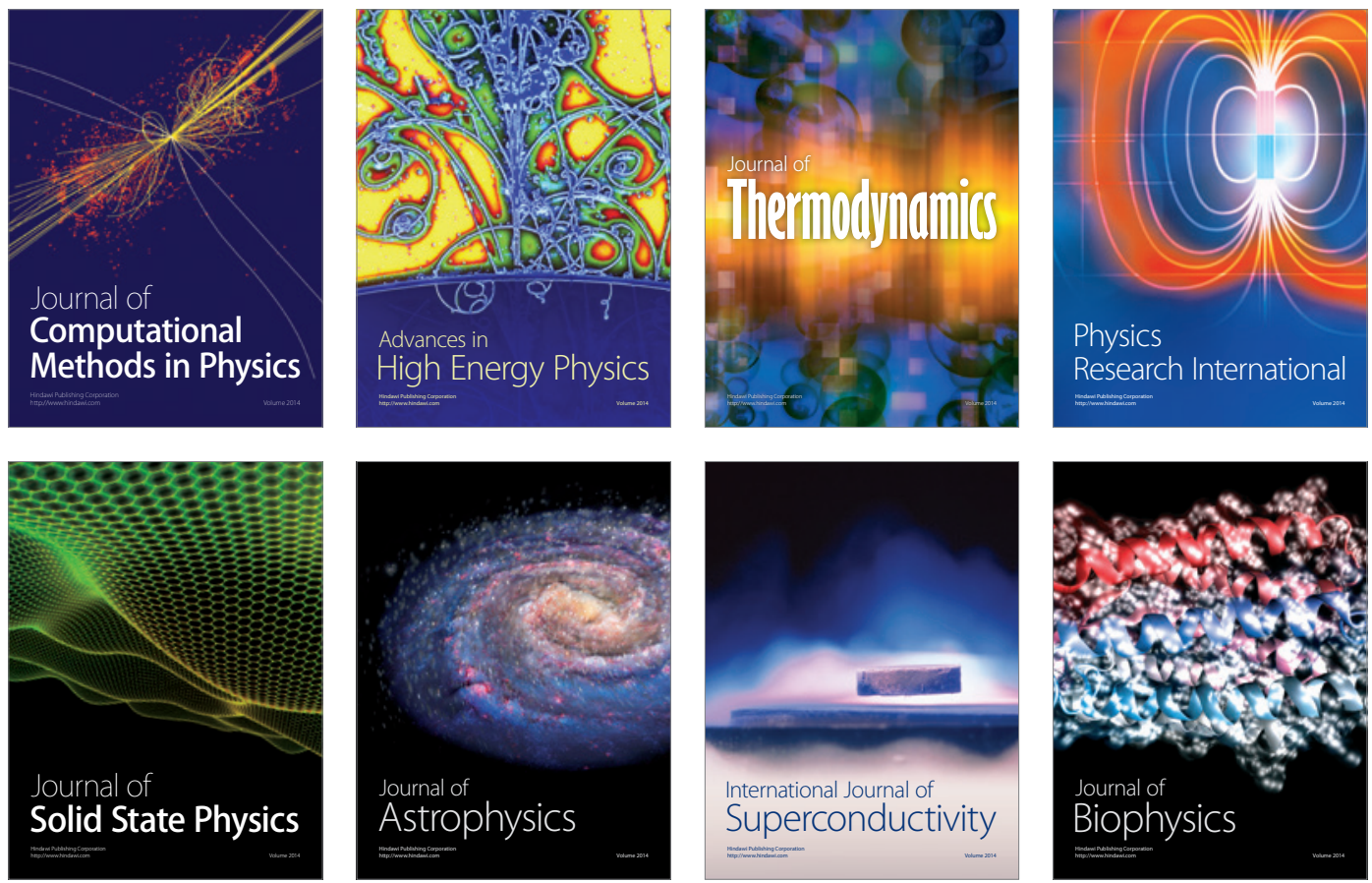
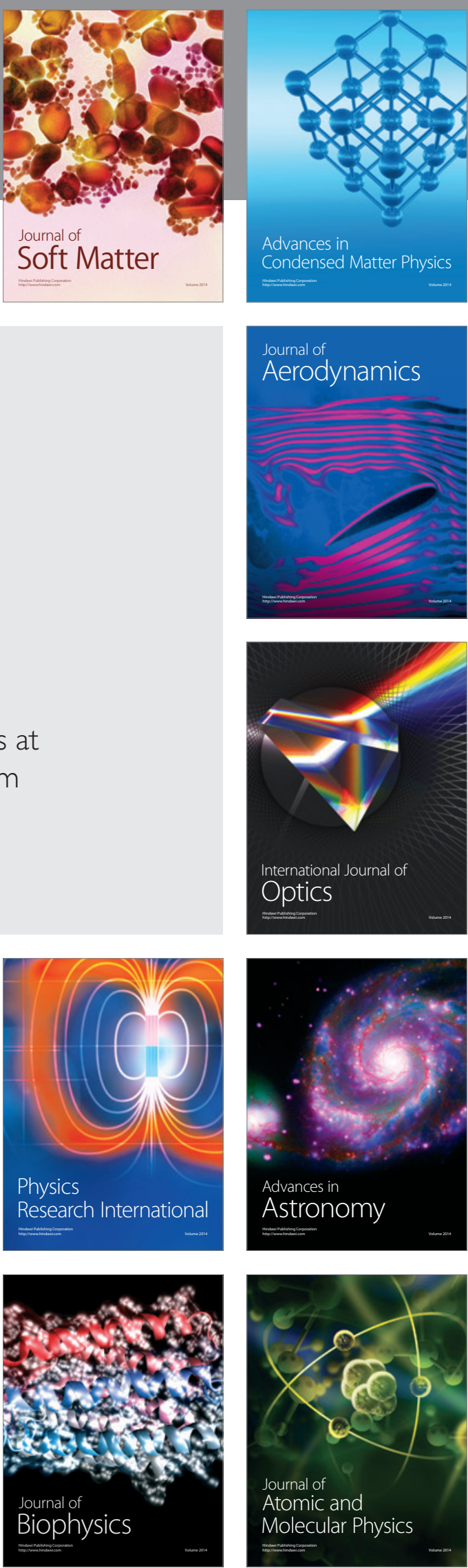